\title{
NSOM LITHOGRAPHY FOR ORGANIZED GROWTH OF GAP NANOWIRES
}

In this contribution, near field scanning optical microscope (NSOM) lithography is presented as a tool for organized growth of nanowires. Non contact mode of NSOM lithography was used to pattern planar structures in photoresist deposited on GaP substrate. In combination with lift-off technique, metal-catalyst particles on GaP substrate for subsequent growth of GaP nanowires were prepared. Different periodic and predefined arrangements of GaP nanowires were achieved.

Keywords: NSOM lithography, predefined structure, organized nanowire growth.

\section{Introduction}

Preparation of semiconductor nanowires (NWs) has been studied intensively due to their unique structural and physical properties that offer big potential for technological applications. Standard technique for one-dimensional growth of NWs is vaporliquid-solid (VLS) mechanism invented in 1960 by Wagner [1]. VLS process starts with formation of a small liquid droplet followed by alloying, nucleation and growth of NW [2]. Growth is started on a clean semiconductor surface. First, metal particles with diameter below $100 \mathrm{~nm}$ are deposited on the substrate surface. Such substrate is heated in reaction chamber until particles melt and form liquid droplets. The semiconductor material is dissolved and forms an alloy which has lower melting point in comparison with pure metal. In this phase, a gas containing growth material flows through the reaction chamber and incorporation of precursor atoms into the alloy leads to supersaturation of semiconductor component. It precipitates at solid-liquid interface which starts growth of NW with diameter determined by size of the droplet [2].

Various technologies have also been used for spatial positioning or organized growth of NWs [2 and 3]. Very convenient is combination of VLS NW growth mechanism with nanopatterning techniques. Patterned metal arrays function as a template; grown NWs have the same pattern, diameter of NWs is correlated to the size of metal particles. Various methods can be applied for controlled preparation of metal template: photolithography [4], e-beam [5], interference [6] or nanosphere lithography [7], as well as manipulation of single gold nanodots [8].

One of optical technologies for planar structure fabrication is near-field scanning optical microscope (NSOM) lithography [9 and 10]. It employs NSOM in illumination mode, where patterning of structures is done through a maskless exposure process performed by the optical near field produced at the tip of scanning fiber probe. Since it is a scanning technique, NSOM lithography is a time-consuming process. On the other hand, this allows wide design flexibility and besides periodic patterns, it allows fabrication of $2 \mathrm{D}$ structures with predefined arrangement. When comparing to different scanning techniques, e. g. e-beam lithography, this technique is low-cost and it doesn't require vacuum environment. In non-demanding experimental setup, exposure of defined regions directly on the device on chip can be achieved. Moreover, due to the exploitation of the near field properties, the spatial resolution limit known from far-field microscopy as a diffraction barrier is overcome; it is no more a function of the wavelength but rather of the fiber tip aperture diameter [11].

We demonstrate NSOM lithography as a tool for organized growth of nanowires. The non-contact mode of NSOM lithography was used to pattern planar structures in photoresist layer deposited in GaP substrate in different two dimensional (2D) grids with pitch step $1.5-2.5 \mu \mathrm{m}$. In this way, predefined metallic nanostructures on $\mathrm{GaP}$ surface which are used for organized $\mathrm{GaP}$ nanowire growth were prepared. $\mathrm{GaP}$ nanowires were grown

\footnotetext{
* ${ }^{1}$ Ivana Lettrichova, ${ }^{1}$ Dusan Pudis, ${ }^{2}$ Agata Laurencikova, ${ }^{2}$ Stanislav Hasenohrl, ${ }^{2}$ Jozef Novak, ${ }^{3}$ Jaroslava Skriniarova, ${ }^{1}$ Peter Gaso ${ }^{1}$ Div. of Optics and Photonics, Dept. of Physics, University of Zilina, Slovakia

${ }^{2}$ Inst. of Electrical Engineering, Slovak Academy of Sciences, Bratislava, Slovakia

${ }^{3}$ Inst. of Electronics and Photonics, Slovak University of Technology, Bratislava, Slovakia

E-mail: lettrichova@fyzika.uniza.sk
} 
by VLS technique using $\mathrm{Au}$ seeds defined by photoresist layer patterning followed by metal evaporation and lift-off technique. Both periodic and predefined 2D arrangements of $\mathrm{GaP}$ nanowires were achieved and analyzed.

\section{Experimental setup}

Schematically is the NSOM lithography process shown in Fig. 1. Experimental stage for NSOM lithography requires high resolution 3D nanoposition system controlled by computer. Because of the photoresist sensitivity in the violet-blue wavelength range, a modulated $473 \mathrm{~nm}$ diode-pumped solid state laser was used as a light source. The laser beam was focused by micro objective into conventional optical fiber, which was coupled to the fiber probe fixed on the 3D axis nanoposition piezosystem. Scanning range of the nanoposition system is $20 \times 20 \mu \mathrm{m}^{2}$ and a movement resolution is less than $5 \mathrm{~nm}$. The non-contact mode of NSOM lithography was performed in this experiment, where the fiber tip realized the in-plane movement over the sample without touching the sample surface.

As a fiber probe, a home-made metal coated apertureless fiber tip was used. It was prepared by pulling of conventional optical fiber in small flame followed by coating by $\mathrm{Al}$ evaporation.

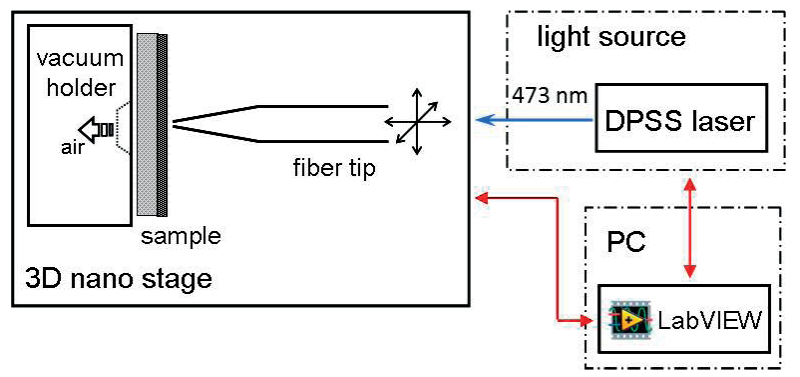

Fig. 1 Experimental set-up for NSOM lithography
GaP NWs were grown on $\mathrm{GaP}(111) \mathrm{B}$ substrate by VLS technique using $\mathrm{Au}$ seeds. For organized growth of NWs, it is necessary to prepare highly ordered and spatially separated metal-catalyst particles. It was achieved using NSOM lithography process in combination with lift-off technique. Schematically, the process is shown in Fig. 2.

First, $500 \mathrm{~nm}$ thin photoresist AZ 5214E was spin-coated on the $\mathrm{GaP}$ substrate with post-baking at $65{ }^{\circ} \mathrm{C}$ for 2 minutes and at $103^{\circ} \mathrm{C}$ for 3 minutes. Photoresist layer was subsequently exposed by NSOM lithography in experimental set-up shown in Fig. 1. After exposure, photoresist was developed in AZ 400K developer for $30 \mathrm{~s}$ and rinsed in deionized water (Fig. 2a). Different periodic and predefined planar structures in grid with pitch step of 1.5 $2.5 \mu \mathrm{m}$ were created in photoresist layer.

In the next step, $2 \mathrm{~nm}$ thick Au layer was evaporated on the top of the sample (Fig. 2b). Lift-off technique was used to remove residual photoresist so that the spatially organized Au seeds were formed on the GaP substrate (Fig. 2c).

NWs were grown in palladium purified $\mathrm{H}_{2}$ carrier gas from phosphine $\left(\mathrm{PH}_{3}\right)$ and trimethylgallium (TMGa) used as the phosphorus and gallium sources, respectively. The doping source was diethylzinc (DEZn). For NWs growth, the GaP substrate with Au seeds was heated at 20 mbar to $650{ }^{\circ} \mathrm{C}$ for 10 minutes under a $\mathrm{H}_{2}+\mathrm{PH}_{3}$ flow with $\mathrm{PH}_{3}$ molar fraction of $1.67 \times 10^{-2}$. Within the next 10 minutes, the pressure was increased to 100 mbar and the temperature was lowered to $500{ }^{\circ} \mathrm{C}$ and stabilized. The TMGa and DEZn flows were finally switched into reactor and the growth of NWs proceeded in VLS mode (Fig. 2d). The molar fractions of $\mathrm{PH}_{3}$ and $\mathrm{TMGa}$ in the carrier gas during the growth were $7.5 \times 10^{-3}$ and $1.25 \times 10^{-5}$, respectively (V/III ratio of 600). After 4 min growth, app. $3 \mu \mathrm{m}$ long GaP NWs were grown.

\section{Results and discussion}

First, 2D structures with periodic and predefined arrangement were prepared in photoresist layer deposited on GaAs substrate. For preparation of 2D structures in GaAs using NSOM lithography, (001) oriented GaAs substrate was used. Exposure

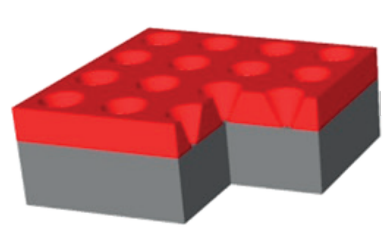

a)

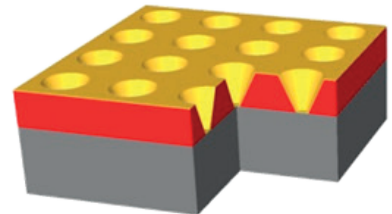

b)

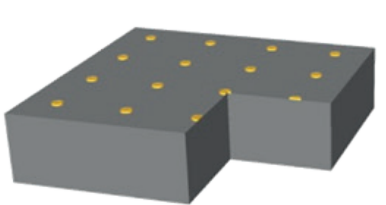

c)

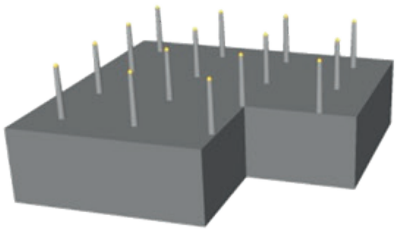

d)

Fig. 2. Fabrication process for organized growth of NWs using NSOM lithography: a) structure patterning in photoresist layer, b) Au coating by evaporation, c) lift-off, creation of organized Au seeds and d) NWs growth by VLS technique. 
by the near field of the fiber tip controlled by PC leads to formation of 2D patterns with desired arrangement.
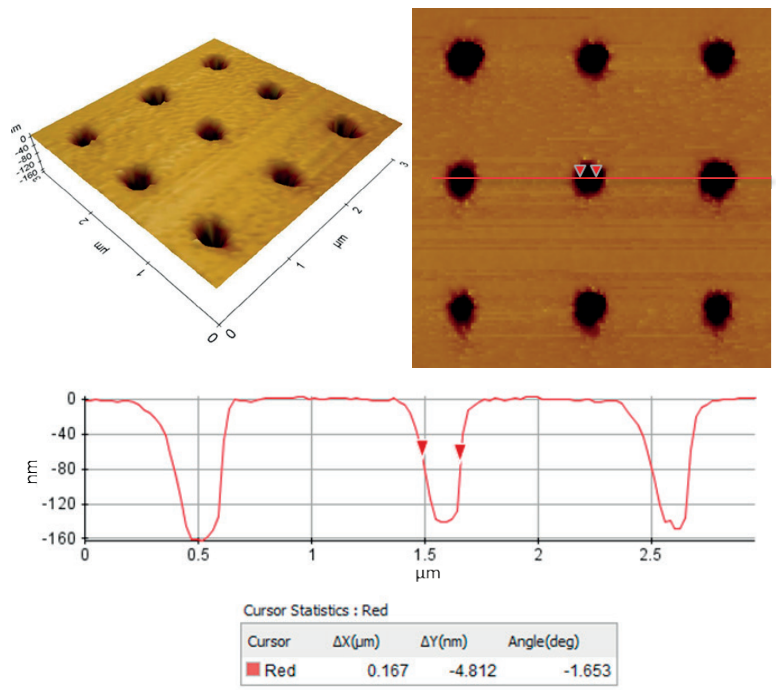

Fig. 3 AFM image and line profile analysis of prepared periodic structure with $1 \mu \mathrm{mm}$ period in both directions and air hole FWHM app. $170 \mathrm{~nm}$

Example of prepared 2D periodic structure is shown in Fig. 3. Air holes were arranged in square lattice with period $1 \mu \mathrm{m}$ in two perpendicular directions. Detail morphology of prepared structure was examined using atomic force microscope (AFM). Line profile analysis documents that full width at half maximum (FWHM) of patterned air holes is app. $170 \mathrm{~nm}$ and the depth is about $160 \mathrm{~nm}$. Due to the nearly Gaussian shape of the exposing optical field irradiated from the fiber probe, air hole edges are oblique, which is in good agreement with published results on NSOM lithography [10]. This characteristic appears inconvenient for etch step, nevertheless, it may be interesting for patterning of metal-catalyst arrays for NWs growth.

NSOM lithography followed by Au evaporation and lift-off technique was used to pattern $\mathrm{Au}$ seeds on $\mathrm{GaP}$ substrate for further organized NW growth. After 4 min growth, groups of app. $3 \mu \mathrm{m}$ long GaP NWs were grown from each Au seed. Different $\mathrm{Au}$ seed arrangements were patterned in the GaP surface. In Fig. 4 , there is shown scanning electron microscope (SEM) image of periodic arrangement that was designed in square lattice with pitch step of $2.5 \mu \mathrm{m}$. Due to big Au particle diameter (about $400 \mathrm{~nm}$ ), GaP NWs were grown radial from the seed and they form a hedgehog-like structure (detail in SEM image in Fig. 4b).

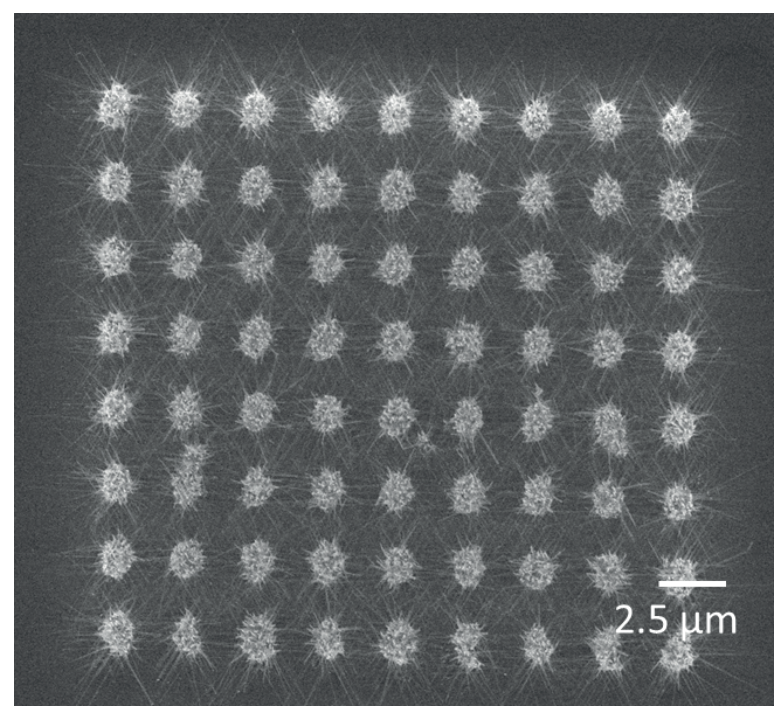

a)

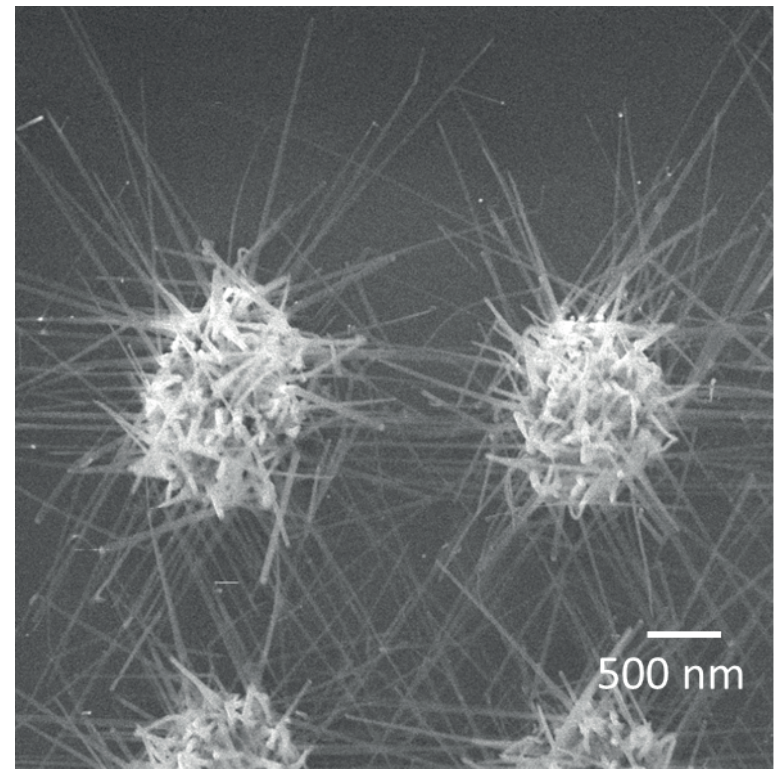

b)

Fig. 4 a) Top-view of prepared organized GaP NWs in regular arrangement and b) detail, SEM images

One of NSOM lithography advantages as scanning technique is possibility to prepare any predefined arrangement of patterned structure. In this way, Au seeds were arranged in different predefined patterns. Example of GaP NWs grown in predefined arrangement is shown in Fig. 5. Heart-shaped structure was designed in grid with $2.5 \mu \mathrm{m}$ pitch step. Due to Au remains in middle part of heart-shaped structure after lift-off technique, GaP NWs were also grown in central unpatterned area. 


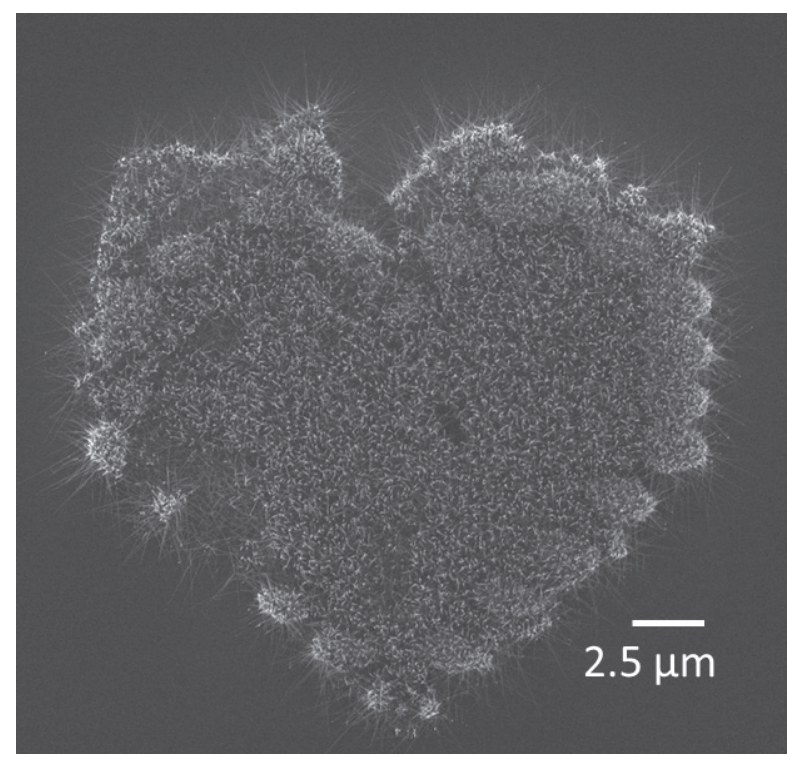

Fig. 5 Top-view of prepared organized GaP NWs in predefined arrangement, SEM image

Diameter and number of metal particles relates to thickness of evaporated $\mathrm{Au}$ layer, as well as to dimensions of photoresist holes formed by lithography process. For NSOM lithography, Gaussian shape of patterned air hole is typical and resolution limits depend on fiber probe apex dimensions [10]. We assume that by fine-tuning of the exposure process, it is possible to reduce photoresist hole diameter down to tens of nanometers in order to decrease number of Au seeds per hole.

\section{Conclusion}

This contribution presents possibilities of non-contact NSOM lithography for organized growth of NWs. In our experimental setup, planar 2D structures with periodic and predefined arrangement with pitch step $1.5-2.5 \mu \mathrm{m}$ were patterned in photoresist layer deposited on GaAs substrate. AFM line profiles document air hole FWHM down to $170 \mathrm{~nm}$.

NSOM lithography was successfully applied as lithography technique for preparation of predefined metallic nanostructures on $\mathrm{GaP}$ surface which were used for organized $\mathrm{GaP}$ nanowire growth. Both periodic and predefined 2D structures were prepared and GaP NWs were grown radial from the seed to form a hedgehog-like structure.

\section{Acknowledgement}

This work was supported by the Slovak National Grant Agency under the projects No. VEGA 1/1058/11 and 1/0528/12 and the Slovak Research and Development Agency under the project No. APVV 0395 12. Authors wish to thank for the support to the R\&D operational program Centre of excellence of power electronics systems and materials for their components II. No. OPVaV-2009/2.1/02-SORO, ITMS 26220120046 funded by European regional development fund (ERDF).

\section{References}

[1] WAGNER, R. S., ELLIS, W. C.: The Vapor-liquid-solid Mechanism of Crystal Growth and its Application to Silicon, Trans. Metall. Soc. AIME 233, 1053-1064, 1965.

[2] FAN, H. J., WERNER, P., ZACHARIAS, M.: Semiconductor Nanowires: From Self-organization to Patterned Growth, Small 2(6), 700-717, 2006.

[3] BARTH, S., HERNANDEZ-RAMIREZ, F., HOLMES, J. D., ROMANO-RODRIGUEZ, A.: Synthesis and Application of One-dimensional Semiconductors, Prog. Mater. Sci. 55, 563-627, 2010.

[4] GREYSON, E. C., BABAYAN, Y. AND ODOM, T. W.: Direct Growth of Ordered Arrays of Small-diameter ZnO Nanowires, Adv. Mater. 16, 1348-1352, 2004.

[5] JENSEN, L. E., BJORK, M. T., JEPPESEN, S., PERSSON, A. I., OHLSSON, B. J., SAMUELSON, L.: Role of Surface Diffusion in Chemical Beam Epitaxy of InAs Nanowires, Nano Lett. 4(10), 1961-1964, 2004.

[6] WEI, Y., WU, W., GUO, R., YUAN, D., DAS, S. AND WANG, Z. L.: Wafer-scale High-throughput Ordered Growth of Vertically Aligned ZnO Nanowire Arrays, Nano Lett. 10(9), 3414 - 3419, 2010.

[7] KOSIOREK, A., KANDULSKI, W., CHUDZINSKI, P., KEMPA, K., GIERSIG, M.: Shadow Nanosphere Lithography: Simulation and Experiment, Nano Lett. 4(7), 1359-1363, 2004. 
[8] OHLSSON, B. J., BJORK, M. T., MAGNUSSON, M. H., DEPPERT, K. SAMUELSON, L., WALLENBERG, L. R.: Size-, shape-, and Position-controlled GaAs Nano-whiskers, Appl. Phys. Lett. 79(20), 3335-3337, 2001.

[9] WEGSCHEIDER, S., KIRSCH, A., MLYNEK, J., KRAUSCH, G.: Scanning Near-field Optical Lithography, Thin Solid Films 264, 264-267, 1995.

[10] KRAUSCH, G., MLYNEK, J.: Surface Modification in the Optical Near Field, Microelectron. Eng. 32, 219-228, 1996.

[11] BETZIG, E., TRAUTMAN, J. K.: Near-field Optics: Microscopy, Spectroscopy, and Surface Modification Beyond the Diffraction Limit, Science 257, 189-195, 1992. 